\title{
Response of P(VDF-TrFE) Sensor to Force and Temperature
}

\author{
Stuart Hannah, Deepak Uttamchandani, Helena Gleskova \\ Department of Electronic and Electrical Engineering, University of Strathclyde, Glasgow, G1 1XW, UK \\ Saleem Khan \\ Fondazione Bruno Kessler, Trento, 38123, Italy \\ Ravinder Dahiya \\ Electronics and Nanoscale Engineering Division, School of Engineering, University of Glasgow, G12 8QQ, UK
}

\begin{abstract}
Responses of a tactile sensor to force (0 to $9 \mathrm{~N})$ and temperature $\left(15\right.$ to $\left.47^{\circ} \mathrm{C}\right)$ are investigated. The sensor is based on ferroelectric polyvinylidene fluoride - trifluoroethylene (P(VDF-TrFE)) copolymer. The 2.5 - $\mu \mathrm{m}$-thick copolymer layer was sandwiched between aluminum and gold electrodes and the active sensing area was $25 \mathrm{~mm}^{2}$. The response of the sensor was measured in a form of capacitance recorded at $2 \mathrm{kHz}$. The capacitance was measured in a steady-state condition, i.e. after the force and temperature have stabilized. We have found that the sensor's sensitivity to temperature is comparable to that of force, and even greatly exceeds the force sensitivity when dealing with forces larger than $3 \mathrm{~N}$. This is because the response of the sensor to temperature is approximately linear, while the response to the applied force is logarithmic. Consequently, at the lower end of applied forces $(<0.5 \mathrm{~N})$, the sensitivity is $7.5 \mathrm{pF} / \mathrm{N}$ while the temperature sensitivity is about $7 \mathrm{pF} /{ }^{\circ} \mathrm{C}$ within the whole temperature range.
\end{abstract}

Keywords - P(VDF-TrFE), Pressure Response, Temperature Response, Capacitance.

\section{INTRODUCTION}

The field of tactile sensing has gained a lot of momentum in recent years, mainly for the development of robotic skin and touch screen interfaces. For example, a humanoid robot being able to detect changes in environmental conditions such as temperature and pressure lends many advantages for exploration of different environmental conditions and for safe human-robot interaction [1]. The existing sensors typically exploit capacitive changes [2,3], piezoresistive effect [4,5] or piezoelectric effects $[6,7]$. The sensor presented in this paper utilizes the ferroelectric copolymer polyvinylidene fluoride trifluoroethylene (P(VDF-TrFE)) that displays piezoelectric and pyroelectric properties $[8,9]$. The piezoelectricity manifests itself as a generation of charge upon application of mechanical stress $[5,10,11]$. By exploiting this effect, large-area sensors can be realized requiring no external power supply [12-15].

In addition to the piezoelectric properties, $\mathrm{P}(\mathrm{VDF}-\mathrm{TrFE})$ also displays a pyroelectric effect when subjected to a temperature gradient [16]. The change in temperature leads to a polarization change within the material and results in the generation of voltage. Consequently, $\mathrm{P}(\mathrm{VDF}-\mathrm{TrFE})$ can be used as both a pressure and temperature sensor $[8,17]$.

978-1-4799-8229-5/15/\$31.00 C2015 IEEE
Previously, $\mathrm{P}(\mathrm{VDF}-\mathrm{TrFE})$ sensor was integrated with a CMOS field-effect transistor to create an all-encompassing sensing and in-situ processing device named piezoelectric oxide semiconductor field-effect transistor (POSFET) [18]. The POSFET features P(VDF-TrFE) layer directly deposited onto the transistor gate, which in turn controls the transistor drain current. To date the POSFET was largely exploited for pressure sensing. This paper compares the response of the $\mathrm{P}(\mathrm{VDF}-\mathrm{TrFE})$ sensor to pressure and temperature to allow the exploitation of POSFET-like devices for temperature monitoring.

This paper is organized as follows: Section II presents development of materials and sensor fabrication process. Measurement procedures are described in section III. Results and discussions are discussed in sections IV and $\mathrm{V}$ respectively. Finally the paper is concluded and future scope is given in section VI.

\section{MATERIALS AND SENSOR FABRICATION}

\section{A. $P(V D F-T r F E)$ Deposition}

A parallel plate structure is developed where the $\mathrm{P}(\mathrm{VDF}-$ TrFE) layer is sandwiched between the two metal plates shown in Fig.1. The bottom contact is made of aluminum, which is thermally evaporated on a clean silicon wafer. The top contact is made of thermally evaporated gold. The completed sensor has an active sensing area of $25 \mathrm{~mm}^{2}$. P(VDF-TrFE) was deposited by spin-coating a 10 wt. \% solution of $\mathrm{P}(\mathrm{VDF}-\mathrm{TrFE})$

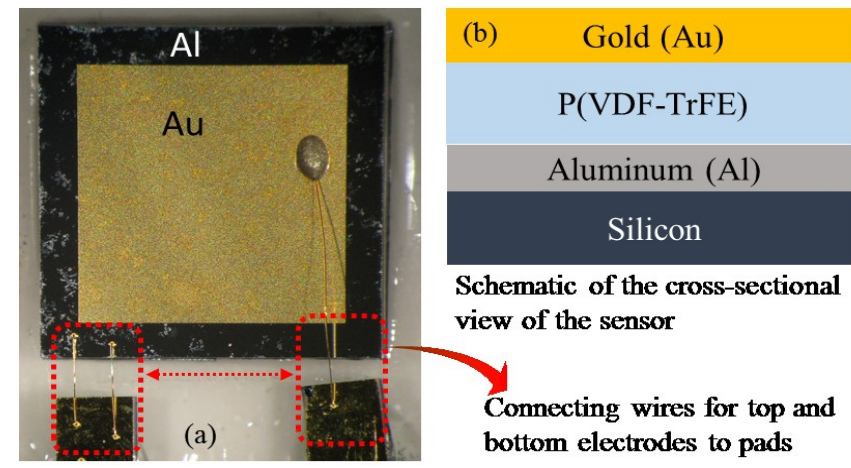

Fig.1. (a) Fabricated sensor where P(VDF-TrFE) is sandwiched between $\mathrm{Al}$ and Au. (b) Schematic of the cross-sectional view of the sensor layers. 
(70:30) copolymer. The 10 wt. \% solution was prepared by mixing $\mathrm{P}(\mathrm{VDF}-\mathrm{TrFE})$ pellets (Piezotech) in RER500 solvent (Fujifilm) and mixing for 4 hours at $80^{\circ} \mathrm{C}$ on a hotplate with magnetic stirrer. Spin-coating was performed in three steps as outlined in [19]. The final P(VDF-TrFE) film thickness was about $2.5 \mu \mathrm{m}$.

\section{B. Annealing Process}

After spin-coating, the P(VDF-TrFE) was annealed at $120^{\circ} \mathrm{C}$ for three hours to remove any excess solvent from the surface and to aid with the crystallization process within the polymer material. $120^{\circ} \mathrm{C}$ was chosen as a suitable temperature as it sits between the $\mathrm{P}(\mathrm{VDF}-\mathrm{TrFE})$ Curie temperature $\left(110^{\circ} \mathrm{C}\right)$ [20] and melting temperature $\left(150^{\circ} \mathrm{C}\right)$ [20] and maximizes the opportunity for the film to crystallize in the $\beta$-phase and thus improve the piezoelectric response of the film [20].

\section{Poling Procedure}

In order to align the dipoles within a piezoelectric material, the process of "poling" is required. Poling is conducted by subjecting the material to a high electric field along a specific direction. The value of electric field required can be lowered if the material temperature is raised, known as "thermal poling" [21]. A temperature below the Curie temperature must be chosen for the poling process otherwise the film's piezoelectric properties will be lost; therefore $80^{\circ} \mathrm{C}$ was used in this case. At this temperature, the electric field of $\sim 80 \mathrm{~V} / \mu \mathrm{m}$ is sufficient to polarize the piezoelectric film. Consequently, a maximum of $240 \mathrm{~V}$ was used for a P(VDF-TrFE) film thickness of $2.5 \mu \mathrm{m}$. Two external metal electrodes were wire bonded to the sensor (see Fig.1). The purpose of these external electrodes is to avoid direct contact of the power supply probes with the surface of the sensors during the poling process, which can deteriorate the thin metal layer. This also provides more space for the characterization tools during force and temperature measurements. There is possibility of sparking when the electric field is increased to the maximum voltage, i.e. $240 \mathrm{~V}$ in this case. To avoid this, the voltage was applied in $40 \mathrm{~V}$ increments and kept for 10 minutes in each stage. Before each voltage increment, the contacts were shorted to mitigate any extra charges which could possibly result in enhancing the sparking and destroying the sandwiched $\mathrm{P}(\mathrm{VDF}-\mathrm{TrFE})$ layer [19].

\section{Measurement Procedures}

Sensor capacitance measurements were performed with Agilent B1500A Semiconductor Parameter Analyzer. The parameter analyzer features a capacitance measurement module capable of measuring at frequencies from $1 \mathrm{kHz}$ to $5 \mathrm{MHz}$ to a resolution of $1 \mathrm{fF}\left(10^{-15} \mathrm{~F}\right)$. Capacitance was extracted at a frequency of $2 \mathrm{kHz}$.

\section{A. Capacitance measurement versus force}

To investigate the sensor's response to pressure, a compressive force was applied in normal direction to the sensor at room temperature via a precision linear stage controlled by a stepper motor (Zaber Technologies). A custom-designed Teflon probe was capable of applying force ranging from $\sim$ few tens of Newton to over $10 \mathrm{~N}$. The contact area of the probe was $2 \mathrm{~mm}$ $\times 6 \mathrm{~mm}$ and allowed applying force across $40 \%$ of the sensor active area.
Sensor capacitance was measured for a range of applied forces from $0 \mathrm{~N}$ to $\sim 9 \mathrm{~N}$. The force was recorded using a load cell (Omega). The capacitance was measured after the force had stabilized. Investigation of change in capacitance was carried out against application of static forces and monitored for possible exploitation of changing capacitance for controlling static events.

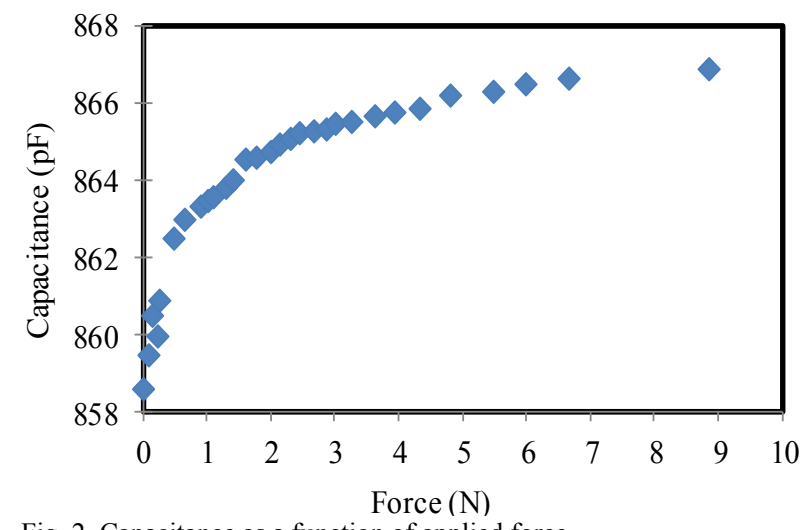

Fig. 2. Capacitance as a function of applied force.

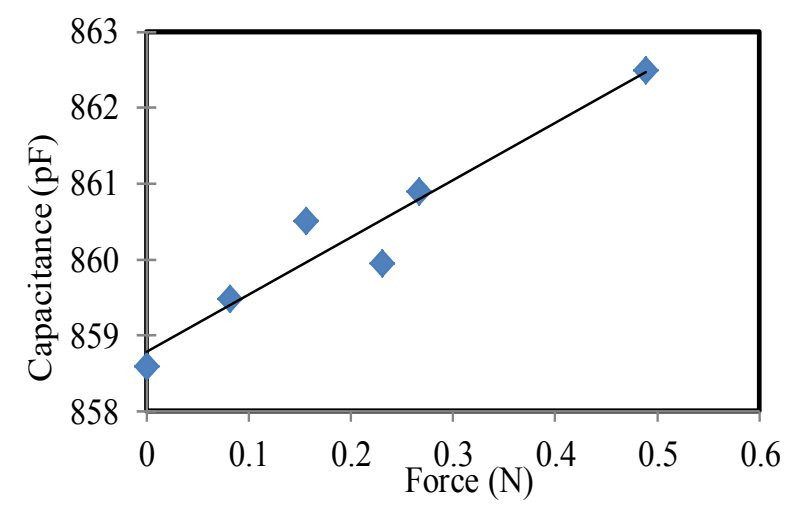

Fig. 3. Capacitance as a function of applied force in the range of 0 to $0.5 \mathrm{~N}$. The slope of the least square linear fit is $7.54 \mathrm{pF} / \mathrm{N}$.

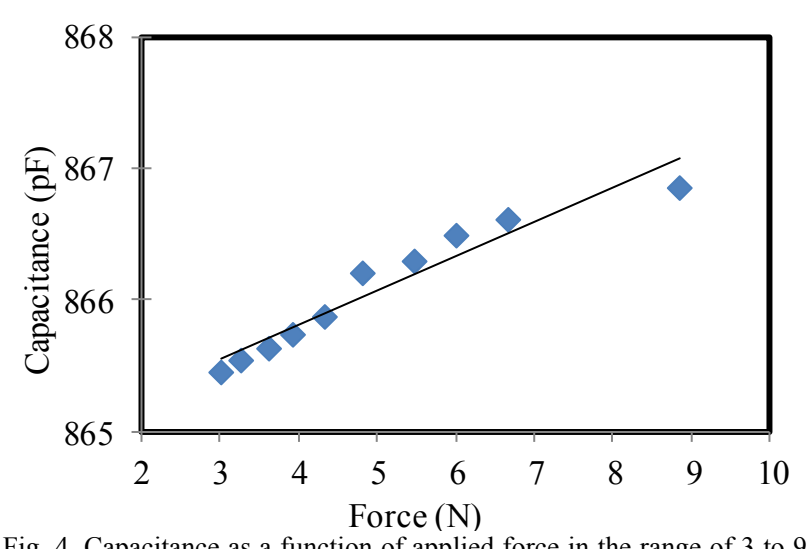

Fig. 4. Capacitance as a function of applied force in the range of 3 to 9 $\mathrm{N}$. The slope of the least square linear fit is $0.26 \mathrm{pF} / \mathrm{N}$.

\section{B. Capacitance measurement versus temperature}

The temperature response of the P(VDF-TrFE) sensor was explored by subjecting it to a changing temperature environment. The temperature was varied between $15^{\circ} \mathrm{C}$ and $47^{\circ} \mathrm{C}$ using a thermoelectric heater/cooler housed in a vacuum chamber with pressure $<10^{-5} \mathrm{mBar}$. The temperature was ramped up and down in $2^{\circ} \mathrm{C}$ increments. The accuracy of the 


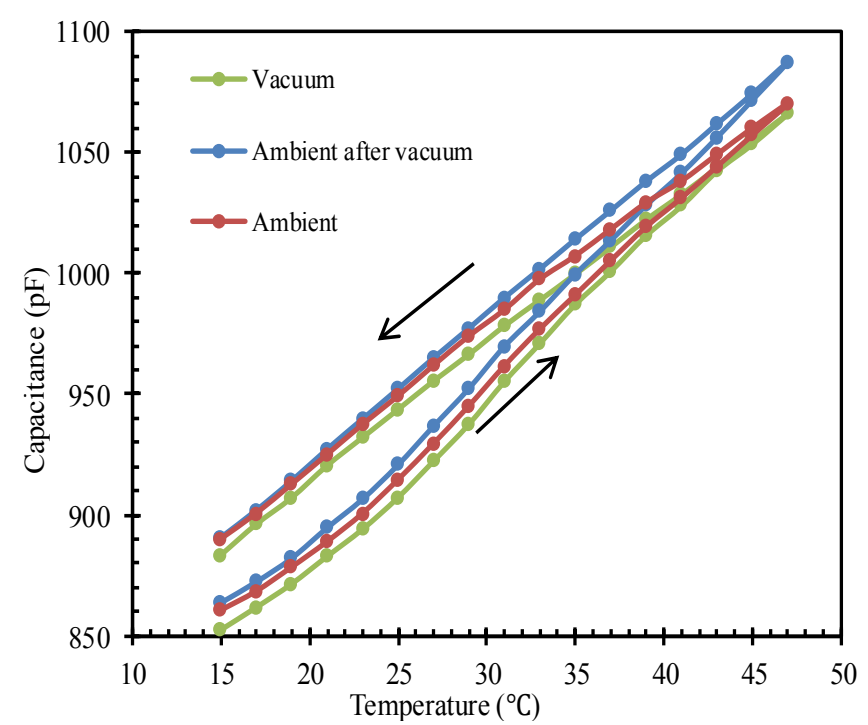

Fig.5. Capacitance as a function of sensor temperature.

temperature measured with thermistor was $\pm 0.5^{\circ} \mathrm{C}$. Capacitance at a frequency of $2 \mathrm{kHz}$ was extracted every $2^{\circ} \mathrm{C}$, after the temperature had stabilized for 5 minutes.

\section{RESULTS}

\section{A. Sensor response against applied force}

Fig. 2 shows the capacitance measurements that resulted in response to applied compressive force carried out on a freshly fabricated sensor at room temperature. The graph shows the change in capacitance from the initial $(0 \mathrm{~N})$ condition, to a maximum force of $8.85 \mathrm{~N}$ applied over a $2 \times 6 \mathrm{~mm}^{2}$ area. At normal conditions when the force is not applied, the initial value of sensor capacitance is $858.59 \mathrm{pF}$. By increasing the applied force to the maximum value of $8.85 \mathrm{~N}$, the sensor capacitance has increased to $866.85 \mathrm{pF}$, leading to the total increase in capacitance of $8.26 \mathrm{pF}$. The capacitance data clearly follows a logarithmic dependence on the applied force. Consequently, the change in capacitance per unit force is much bigger for low force when compared to high force.

Fig. 3 shows the sensor capacitance extracted for lower forces between $0 \mathrm{~N}$ and $\sim 0.5 \mathrm{~N}$. The sensor sensitivity for this force range is $\sim 7.5 \mathrm{pF} / \mathrm{N}$. Fig. 4 includes the capacitance data for forces at the higher end of the scale (3-9 N). Once again, this force range shows approximately a linear dependence; however, the sensitivity in this region is vastly reduced compared to that of Fig. 3. The sensitivity in this case is $\sim 0.26 \mathrm{pF} / \mathrm{N}$.

\section{B. Sensor response against changing temperature}

The effect of ambient temperature on sensor capacitance was investigated in accordance with the experimental procedure outlined in section 3. Three experiments were performed to establish whether humidity/moisture affected the sensor capacitance during the temperature variations. The experiments were carried out on the same sensor, several days apart, to allow the recovery of the P(VDF-TrFE) sensor between the measurements. The initial capacitance measured at room temperature in each case was $\sim 875 \mathrm{pF}$ prior to the application of the temperature ramp. The three capacitance/temperature experiments were as follows:
TABLE 1. Temperature sensitivity of sensor for various conditions.

\begin{tabular}{|cl|l|}
\hline \multicolumn{2}{|c|}{ Experiment } & Sensitivity $\left(\mathbf{p F}{ }^{\circ} \mathbf{C}\right)$ \\
\hline 1$)$ & Vacuum & 7.04 \\
\hline 2$)$ & Ambient after vacuum & 7.22 \\
\hline 3$)$ & Ambient & 6.87 \\
\hline
\end{tabular}

1) Capacitance measured in vacuum $\left(<10^{-5} \mathrm{mBar}\right)$ after keeping the sensor in low vacuum for three days

2) Capacitance measured in ambient air after keeping the sensor in low vacuum for four days

3) Capacitance measured in ambient air after keeping the sensor in ambient air for one week

Fig. 5 shows the capacitance measured for all three experiments conducted as a function of sensor temperature. Capacitance was measured with no force applied on the sensor to ensure any change in sensor capacitance is related to temperature. Fig. 5 shows the change in capacitance as temperature was ramped up and down. In all three cases, the capacitance responds approximately linearly to the change in temperature. In addition, small hysteresis is present in the sensor behavior.

Table 1 summarizes the extracted temperature sensitivities for the three measurement conditions. Experiment 2 (capacitance measured in ambient air after storage in vacuum) leads to the greatest temperature sensitivity. The least sensitive condition is storage and measurement in ambient air. However, all temperature sensitivities are the same within $5 \%$. In summary, all measurement conditions display similar sensitivities of $\sim 7 \mathrm{pF} /{ }^{\circ} \mathrm{C}$ with linear responses within the temperature range explored.

\section{DISCUSSION}

The sensor capacitance shows a logarithmic dependence on applied force over the investigated force range up to $9 \mathrm{~N}$ Forces below $0.5 \mathrm{~N}$ show a much greater sensitivity $(\sim 7.5$ $\mathrm{pF} / \mathrm{N})$ than forces between 3 and $9 \mathrm{~N}(\sim 0.26 \mathrm{pF} / \mathrm{N})$. High sensitivity at the lower force range is important, as the majority of manipulative tasks carried out by a humanoid robot during day to day activities are in the region of $<1 \mathrm{~N}$ force [18].

Conversely, the sensor capacitance varies approximately linearly with changing temperature in the range of 15 to $47^{\circ} \mathrm{C}$. The sensitivity is about $7 \mathrm{pF} /{ }^{\circ} \mathrm{C}$, regardless of the sensor history and the measurement condition. Consequently, the sensor could be used as an effective temperature sensor.

The change in sensor capacitance as a function of temperature $\left(\sim 7 \mathrm{pF} /{ }^{\circ} \mathrm{C}\right)$ for all three conditions studied is comparable with that of force $(7.5 \mathrm{pF} / \mathrm{N})$ for forces $<0.5 \mathrm{~N}$. We therefore propose that this $\mathrm{P}(\mathrm{VDF}-\mathrm{TrFE})$ tactile sensor could be explored for temperature sensing in addition to its current application for pressure/touch sensing [18]. Previously a p-n junction temperature diode was combined with POSFET to allow temperature monitoring [12]. The output was linear with sensitivity of $2.36 \mathrm{mV} /{ }^{\circ} \mathrm{C}$. However, using a separate diode component complicates the chip, when the same fabrication procedure could be used to manufacture both pressure and temperature sensors, or with complex signal processing, a single sensor could be used as a multi-functional pressure and temperature sensor, drastically reducing the 
amount of space taken up on a chip and lowering the overall fabrication cost.

The capacitance data displays small hysteresis as the temperature is ramped back down from the maximum. Further investigation into the origin of the hysteresis needs to be done in the future.

\section{CONCLUSION}

Response of $\mathrm{P}(\mathrm{VDF}-\mathrm{TrFE})$ tactile sensor to force and temperature was investigated in a comparative study. The force in the range of 0 to $9 \mathrm{~N}$ and temperatures between $15^{\circ} \mathrm{C}$ and $47^{\circ} \mathrm{C}$ were applied. The response of the sensor was measured in a form of capacitance recorded at $2 \mathrm{kHz}$. The capacitance was measured in a steady-state condition, i.e. after the force and temperature had stabilized. We have identified that the sensitivity of the $\mathrm{P}(\mathrm{VDF}-\mathrm{TrFE})$ sensor to temperature is comparable or higher than its sensitivity to the applied force. This is because the response of the sensor to temperature is approximately linear, while the response to the applied force is logarithmic. Consequently, at the lower end of applied forces $(<0.5 \mathrm{~N})$, sensitivity is $7.5 \mathrm{pF} / \mathrm{N}$ while the temperature sensitivity is $\sim 7 \mathrm{pF} /{ }^{\circ} \mathrm{C}$ regardless of the sensor history and the measurement condition. Consequently, the P(VDF-TrFE) sensor could be used for temperature sensing in addition to its wide use as a pressure/touch sensor.

Looking forward, the capacitance change seen via the temperature change is significant enough to allow the sensor to be connected to the gate of a thin-film transistor in order to modulate transistor drain current, creating a fully flexible, lowcost sensing and processing system to record changes in temperature accurately.

\section{ACKNOWLEDGMENT}

We thank Li Ma for help with temperature measurements, and Alan Davidson and Ivan Glesk for help with the experimental design and setup. Stuart Hannah acknowledges support from the Engineering and Physical Sciences Research Council (EPSRC). We also thank colleagues from Microsystem Technology groups at FBK, Italy, for their support related to $\mathrm{P}(\mathrm{VDF}-\mathrm{TrFE})$ samples.

\section{REFERENCES}

[1] N. Elkmann, M. Fritzsche, and E. Schulenburg, "Tactile sensing for safe physical human-robot interaction," in ACHI 2011, The Fourth Int. Conf on Advances in Computer-Human Interactions, 2011, pp. 212-217.

[2] J. Han and M. A. Shannon, "Smooth contact capacitive pressure sensors in touch-and peeling-mode operation," IEEE Sensors J., vol. 9, pp. 199206, 2009.

[3] E. G. Bakhoum and M. H. Cheng, "Novel capacitive pressure sensor," Microelectromechanical Systems, Journal of, vol. 19, pp. 443-450, 2010.

[4] J. Park, Y. Lee, S. Lim, Y. Lee, Y. Jung, H. Lim, et al., "Ultrasensitive Piezoresistive Pressure Sensors Based on Interlocked Micropillar Arrays," BioNanoScience, vol. 4, pp. 349-355, 2014.

[5] S. Khan, R. S. Dahiya, and L. Lorenzelli, "Bendable Piezoresistive Sensors by Screen Printing MWCNT/PDMS Composites on Flexible Substrates," in 10th Int. Conf. on Ph.D Res. in Microelectr. and Electr., Grenoble, France, 2014.

[6] L. Wang, L. Qin, and L. Li, "Piezoelectric dynamic pressure sensor," in Information and Automation (ICIA), 2010 IEEE International Conference on, 2010, pp. 906-911.

[7] A. Kimoto and S. Shimada, "A Proposal of New Multifunctional Pressure Sensor Based on PVDF Films," IEEE Trans. Instrumentation and Measurement, , vol. 62, pp. 2870-2877, 2013.
[8] B. Ploss, B. Ploss, F. G. Shin, H. L. Chan, and C. Choy, "Pyroelectric or piezoelectric compensated ferroelectric composites," Applied Physics Letters, vol. 76, pp. 2776-2778, 2000.

[9] G. Bai, R. Li, Z. Liu, Y. Xia, and J. Yin, "Tuned dielectric, pyroelectric and piezoelectric properties of ferroelectric P (VDF-TrFE) thin films by using mechanical loads," JJ. Appl. Phys., vol. 111, p. 044102, 2012.

[10] M. Oshiki and E. Fukada, "Piezoelectric effect in stretched and polarized polyvinylidene fluoride film," Japan. J. Appl. Phys., vol. 15, p. 43, 1976.

[11] S. Khan, S. Tinku, L. Lorenzelli, and R. S. Dahiya, "Conformable Tactile Sensing using Screen Printed P(VDF-TrFE) and MWCNTPDMS Composites " in IEEE-Sensors, Valencia, Spain, 2014, pp. 1-4.

[12] R. S. Dahiya and M. Valle, Robotic tactile sensing: technologies and system: Springer Science \& Business Media, 2012.

[13] S. Khan, R. S. Dahiya, and L. Lorenzelli, "Screen Printed Flexible Pressure Sensors Skin," in ASMC 2014 - The 25th Annual Adv. Semiconductor Manufacturing Conf., NY, USA, 2014, pp. 219-224.

[14] C. Li, P. M. Wu, S. Lee, A. Gorton, M. J. Schulz, and C. H. Ahn, "Flexible Dome and Bump Shape Piezoelectric Tactile Sensors Using PVDF-TrFE Copolymer," J. Microelectromech. Sys., vol. 17, pp. 334341, 2008.

[15] S. Khan, S. Tinku, R. S. Dahiya, and L. Lorenzelli, "Flexible Tactile Sensors using Screen Printed P(VDF-TrFE) and MWCNT/PDMS Composites," Sensors Journal, IEEE, vol. PP, pp. 1-1, 2014.

[16] A. Bune, C. Zhu, S. Ducharme, L. Blinov, V. Fridkin, S. Palto, et al., "Piezoelectric and pyroelectric properties of ferroelectric LangmuirBlodgett polymer films," J. Appl. Phys, vol. 85, pp. 7869-7873, 1999.

[17] B. Ploss, F. Shin, H. Chan, and C. Choy, "Pyroelectric activity of ferroelectric PT/PVDF-TRFE," Dielectrics and Electrical Insulation, IEEE Transactions on, vol. 7, pp. 517-522, 2000.

[18] R. Dahiya, G. Metta, and M. Valle, "Piezoelectric polymer oxide semiconductor field effect transistor (POSFET) devices for touch sensing," in IEDST'09. 2nd International Workshop on Electron Devices and Semiconductor Technology, 2009, pp. 1-5.

[19] R. S. Dahiya, M. Valle, G. Metta, L. Lorenzelli, and S. Pedrotti, "Deposition, processing and characterization of $\mathrm{P}(\mathrm{VDF}-\mathrm{TrFE})$ thin films for sensing applications," in 2008 IEEE Sensors, 2008, pp. 490-493.

[20] K. Lau, Y. Liu, H. Chen, and R. Withers, "Effect of Annealing Temperature on the Morphology and Piezoresponse Characterisation of Poly (vinylidene fluoride-trifluoroethylene) Films via Scanning Probe Microscopy," Advances in Condensed Matter Physics, vol. 2013, 2013.

[21] H. S. Nalwa, Ferroelectric Polymers: Chemistry: Physics, and Applications vol. 28: CRC Press, 1995. 